\title{
Utilization And Effectiveness Of Malaria Prevention Methods Among Pregnant Women In A Rural Community Of Ekiti State, South West Nigeria: A Cross- Sectional Study.
}

Azeez Ibrahim ( $\sim$ ibrahimazeez1434@gmail.com )

Federal Teaching Hospital, Ido Ekiti

\section{Tosin Anthony Agbesanwa}

Ekiti State University College of Medicine

Olabode Muftau Shabi

Afe Babalola University College of Medicine and Health Sciences

Kayode Ebenezer Ariyibi

Federal Teaching Hospital, Ido Ekiti

Ayodele Kamal Alabi

Federal Teaching Hospital, Ido Ekiti

Oluwatosin Adejumoke Omonijo

Federal Teaching Hospital, Ido Ekiti

\section{Research Article}

Keywords: Malaria, pregnant wo men, malaria prevention methods, rural community, Ekiti State.

Posted Date: November 18th, 2020

DOl: https://doi.org/10.21203/rs.3.rs-110380/v1

License: (c) (i) This work is licensed under a Creative Commons Attribution 4.0 International License.

Read Full License 


\section{Abstract}

Background: National surveys indicate that among countries in Africa where malaria is endemic, Nigeria alone accounts for $21 \%$ of pregnancies that are unprotected from malaria. The purpose of this study is to ascertain the extent of utilization and effectiveness of malaria prevention methods, to find out the existing relationship between maternal parasitaemia and the effectiveness of these methods at $(p<0.05)$ level of significance.

Methods: The study was a cross sectional survey involving 264 consenting booked pregnant women who were recruited from antenatal clinic at Federal Teaching Hospital, Ido -Ekiti, Ekiti State, Southwestern Nigerian. Interviewer administered questionnaire was used to elicit information on the socio-demographic characteristics of the respondents, mode of malaria prevention, extent of utilization of malaria prevention methods and their effectiveness. Venous blood samples for malaria parasite test were collected and microscopic analysis was done to detect the malaria parasite in the obtained blood samples. Data was analyzed using SPSS version 20.0

Results: All the 264 pregnant women interviewed, consented to having their blood taken. In rank order, the mode of malaria prevention methods most commonly reported as being effective were Intermittent Preventive Treatment using sulphadoxine-pyrimethamine (IPT-sp) which was favored by majority 190 (72.0\%). This was followed by Insecticide Spray 166 (62.9\%). Insecticide Treated Nets (ITNs) were the least utilized by $108(40.9 \%)$ respondents. Nine (8.3\%) of the 108 participants that used ITNs, 15 (7.9\%) of the 190 participants that used IPT-s and $18(10.8 \%)$ of the 156 participants that used Insecticide Spray tested positive for malaria respectively. There was a statistically significant association between maternal parasitaemia and malaria prevention methods using ITNs $(p=0.001)$, IPT-sp $(p=0.001)$ and Insecticide sprays $(p=0.001)$.

Conclusion: The three malaria preventive methods adopted for this study were ITNs, IPT-sp and Insecticide Spray. Despite their protective effectiveness, their utilizations are still poor. Information and communication strategies with other concerted efforts by the stakeholders are suggested to ensure proper implementation of the national policy on malaria as adopted by other countries.

\section{Introduction}

Malaria infection is caused by Plasmodium species, most especially Plasmodium falciparum in our environment and is transmitted by female anopheles mosquitoes. Plasmodium falciparum accounts for over $80 \%$ of the world malaria burden [1]. The other three species (P.vivax, P.ovale and P. malariae) have contributed little or no impact to the burden of malaria in pregnancy especially in sub-Saharan Africa [1]. Malaria is the leading cause of anaemia in pregnancy and accounted for 10,000 and 200,000 maternal and neonatal deaths per year respectively [2]. Approximately, 25 million women living in malaria endemic areas of sub-Saharan Africa become pregnant every year. In Nigeria, about 8 million pregnant women are at risk of malaria during pregnancy $[1,2]$. The impact of malaria infection in pregnancy is due to placenta 
and peripheral blood parasitaemia causing maternal anaemia and low birth weight $[2,3]$.

Malarial symptoms in pregnancy differ with the endemicity of its transmission and the level of immunity acquired by the pregnant women [2,3] High grade fever, anaemia, hypoglycaemia, and other complications of malaria in pregnancy can cause grave consequences to the pregnant mothers and the unborn babies [2, 4]. These adverse effects of malaria made the World Health Organization (WHO) developed three approaches for its control in pregnancy [5]. These include the use of Insecticide Treated Nets (ITNs), Intermittent Preventive Treatment using Sulphadoxin - pyrimethamine (IPT- $s p$ ) and prompt access to effective case management [5]. Although, many of the African countries including Nigeria have started the implementation of ITNs usage, IPT-sp and prompt case management, only $20 \%$ of the pregnant mother received good preventive regimen with the situation being worse among pregnant women living in rural areas [5]. The utilization rate of insecticide treated nets has been studied by researchers with mixed findings[6-8]. The national estimates of the proportion of pregnant women who slept under ITNs the night before survey was conducted was 33.7\% [6]. The comparative utilization rate across Nigeria are ; $28.5 \%, 31.6 \%$ in Ibadan (Southwestern Nigeria) [7, 8], 25.7\% in Calabar(SouthSouthern Nigeria)[9], 39.1\% in Enugu [10], 41.0\% in Oweri (South Eastern Nigeria)[11], and 57.0\% in Sokoto(Northwestern Nigeria)[12]. The effectiveness of malaria prevention methods in preventing adverse outcomes from maternal parasitaemia has also been studied by several researchers [6-8]. When used in pregnancy, ITNs have been reported in metal- analyses to reduce placental parasitaemia by $23 \%$ [13]. The use of two or three doses of IPTs-sp in pregnancy has been shown to significantly reduce the risk of malaria parasitaemia $[13,14]$. In Nigeria and other developing countries, there are other approaches that pregnant women often use as protective measures against malaria in their localities [10]. These could be insecticide sprays, mosquitoes repellent creams, window net among others [10]. Previous studies by Tongo et al [15] and Michael et al [16] have confirmed the effectiveness of insecticide spray against malaria parasitaemia in pregnancy.

Since year 2000 when Nigeria adopted the use ITNs and IPT-sp for malaria control as National policy, several researchers have reported the low utilization of these strategies. Available literatures on the utilization and effectiveness of malaria prevention methods in health institutions in Nigeria have been restricted to centres in urban areas. However, the rural area is where malaria mostly threatens the life of many pregnant women and their unborn babies. This study aimed to address this gap. The objectives of this study, therefore, are:

1. To determine the utilization of the commonly used malaria prevention methods;

2. To ascertain the effectiveness of these methods against malaria infection among pregnant women attending a tertiary health facility in a rural community of Ekiti State, Southwestern Nigeria.

3. To identify any existing relationship between malaria infection rate and malaria prevention method utilization.

The study answered the following research questions: 
1. What is the extent of utilization of malaria prevention methods as adopted by the study participants?,

2. What is the level of protective effectiveness of malaria prevention methods as adopted by the study participants?.

The following null hypothesis was tested at 0.05 level of significance:

There is no statistically significant relationship between malaria infection rate and malaria prevention methods.

\section{Materias And Methods}

\section{Study area}

The study was carried out between April and October 2019 at Igogo Ekiti Outreach of Federal Teaching Hospital( FTH), Ido-Ekiti, Ekiti State, Nigeria. Igogo- Ekiti is in Moba Local government area and about $50.5 \mathrm{~km}$ from Ado-Ekiti, the state capital. Ekiti State has tropical climate with two distinct seasons; the rainy season (April-October) and the dry season (November - March). The full description of the study area was in our recent study [17].

\section{Study design}

The study was an hospital-based cross sectional analytical survey.

\section{Study Population}

The study population was all pregnant women who presented for antennal care at Igogo outreach centre of the FTH, Ido -Ekiti, Ekiti State.

\section{Inclusion criteria}

All pregnant women aged 18 years and above, who presented for antenatal care and consented to the study were included.

\section{Exclusion criteria}

Women with pregnancy complications, those currently on malaria treatment or have just completed antimalaria drug within the last two weeks were excluded.

\section{Sample size determination}

Sample size was determined using the formula[18]; 
$\mathrm{n}=\mathrm{Z}^{2} \mathrm{p}(\mathrm{P}-\mathrm{P} 1)$ and $\mathrm{nf}=\underline{\mathrm{n}}$

$$
d^{2} \quad 1+n / N
$$

Where $\mathrm{n}=$ the minimum sample size when population is more than 10,000 and $\mathrm{nf}$ is the minimum sample size when the population is less than $10,000, \mathrm{~N}$ is the estimate of population size, $\mathrm{Z}$ is the standard normal deviate set at 1.96 which corresponds to $95 \%$ confidence interval, $d$ is the desire level of precision set at 0.05 level, $P$ is the estimated proportion with the attribute of interest which is $23 \%$ [19]. The calculated sample size of 264 was used for this study after adding $10 \%$ attrition rate to cover for unexpected data losses.

\section{Sampling technique and recruitment procedure}

A systematic random sampling was employed to recruit the 264 participants who fulfilled the inclusion criteria. The medical record of pregnant women who presented for antenatal care in the year 2018 showed that an average of 40 pregnant women booked weekly and 520 estimated participants booked over a 13- weeks period of recruitment. Using sample interval $K=N / n, K=520 / 264=2$. Therefore, every 2nd participant was selected by systematic random sampling after selecting the first participant by simple random on each recruitment day. A sticker was placed on each of the selected file to avoid resampling at the subsequent clinics.

\section{Data quality assurance}

The data was collected using a pretested semi- structured interviewer administered questionnaire. The questionnaire was validated by subjecting to face and content validity. The reliability of the instrument was determined using cronbach alpha and a reliability coefficient of 0.81 was obtained which was within acceptable limits. To ensure construct validity, the instrument was pretested on 15 pregnant women who were selected randomly at the antennal clinic of the Ekiti State University Teaching Hospital, Ado -Ekiti. Feedback from the pretest and validity assessement led to some adjustment in the questionnaire. The questionnaire was translated from English to Yoruba language for those who did not understand English with the help of an interpreter. Back translation to English was subsequently done to maintain response consistency. It took about 10 minutes to complete the questionnaire. The methods and objectives of the study were carefully explained to the participants individually, after which written informed consent was obtained either by appending signature or thumbprint for consent to participate in the study. Anonymity and confidentiality of the participants were assured, while participants were told that participation in the study was voluntary and non-participation would in no way affect their treatment or care during the antenatal clinic.

\section{Data collection procedure:}


The questionnaire elicited information on the participants socio-demographic characteristics, the utilization of ITNs, insecticide sprays and IPT-sp. Clinical thermometer was used to record the temperature of each participant. Quick general and abdominal examinations were done.

\section{Microscopy}

About $3 \mathrm{mls}$ of venous blood sample was collected from each participant using sterile needle and syringe after consent has been sought and given. The blood sample was transferred into anti-coagulant EDTA bottle. The bottle was labeled to match the participant identity number and the number on the questionnaires before being sent to the laboratory. Two blood films, thin and thick were prepared from the sample on glass slide (blood smear) for malaria parasitaemia. The smears were stained with $2 \%$ Giemsa stains BDH Laboratory supplies, Poole BH 15 ITD England, [19] and then examined under a microscope at x100 magnification by two independent medical laboratory scientists working in the hospital and who were blinded to the result of each other. In case there was a discordance in the result from the two scientists, a senior scientist was recruited to re-examine the slide for approval. Quality control was ensured by the senior scientist.

\section{Data Analysis}

The Statistical Packaged for Social Sciences (SPSS) for window software version 20.0 was used to analyze athe data collected. The socio-demographic characteristics of the participants, the body temperature and the utilization of malaria prevention methods were described using appropriate tables and charts. Chi-square analysis was used to determine the significance of association. Mean value was compared by student-t-tests. The chi-square test and Fisher's exact test were used to compare proportion of categorical variables. A p-value" of $<0.05$ was accepted as statistically significant.

\section{Results}

Two hundred and sixty four pregnant women were studied. The mean age of the participants (Table 1) was $29.1 \pm 5$ (range:18-44) years. Virtually, all the participants (99.2\%) were married. Majority (94.3\%) were rural dwellers, over half (54.2\%) were skilled workers while most of them (78.8\%) had primary school level of education and $76.9 \%$ were from monogamous family. More than half of them $(60.2 \%)$ had family size of five or less. Participants were majorly (92.0\%) of low socio-economic status. 
Table 1: Respondents' demographic characteristics $(N=$ 264)

\begin{tabular}{|c|c|c|}
\hline Variables & Frequency(n) & $\begin{array}{l}\text { Percent } \\
\text { (\%) }\end{array}$ \\
\hline \multicolumn{3}{|l|}{ Age group (years) } \\
\hline Less than 25 yrs & 51 & 19.3 \\
\hline $25-29 \mathrm{yrs}$ & 83 & 31.4 \\
\hline $30-34$ yrs & 95 & 36.0 \\
\hline 35 yrs and above & 35 & 13.3 \\
\hline$M e a n \pm S D($ years $)$ & $29.1 \pm 5.0$ & \\
\hline \multicolumn{3}{|l|}{ Domicile } \\
\hline Urban Ekiti & 15 & 5.7 \\
\hline Rural Ekiti & 249 & 94.3 \\
\hline \multicolumn{3}{|c|}{ Respondent's occupation } \\
\hline Professionals, etc & 10 & 3.8 \\
\hline Skilled & 143 & 54.2 \\
\hline Unskilled & 111 & 42.0 \\
\hline \multicolumn{3}{|c|}{ Respondent's education } \\
\hline Tertiary & 4 & 1.5 \\
\hline Secondary & 29 & 11.0 \\
\hline Primary & 208 & 78.8 \\
\hline None & 23 & 8.7 \\
\hline \multicolumn{3}{|l|}{ Marital status } \\
\hline Single & 2 & 0.8 \\
\hline Married & 262 & 99.2 \\
\hline \multicolumn{3}{|l|}{ Type of family } \\
\hline Monogamous & 203 & 76.9 \\
\hline Polygamous & 61 & 23.1 \\
\hline \multicolumn{3}{|l|}{ Socio-economic status } \\
\hline High & 1 & 0.4 \\
\hline Middle & 20 & 7.6 \\
\hline Low & 243 & 92.0 \\
\hline
\end{tabular}

Most (72.2\%) of the participants (Fig. 1) used IPT-sp alone, 166 (62.9\%) used insecticide spray alone, while 108 (40.9\%) used ITNs alone, 134 (50.8\%) used insecticide spray and IPT-sp, 89 (37.7\%) used ITNs and IPT-sp, 80 (30.3\%) used insecticide spray and ITNs and $69(26.1 \%)$ used all the three strategies.

As shown in Table 2, the body temperature of majority of the participants 230 (87.1\%) ranged from 36.537.2 degree Celsius. 
Table 2

Temperature levels in participants

\begin{tabular}{|lll|}
\hline Temperature $\left({ }^{\circ} \mathrm{C}\right)$ & Frequency(n) & Percent(\%) \\
\hline$<36.5$ & 171 & 64.8 \\
\hline $36.5-37.2$ & 66 & 25.0 \\
\hline$>37.2$ & 27 & 10.2 \\
\hline Total & 264 & 100.0 \\
\hline
\end{tabular}

Nine of the 108 participants (Table 3) that used ITNs, 15 of the 190 participants that used IPT-sp and 18 of the 156 participants that used Insecticide Spray were tested positive for malaria parasitaemia.

Therefore, the prevalence of malaria parasitaemia by ITNs, IPT-sp and Insecticide Spray was 8.3\%, 7.9\% and $10.8 \%$ respectively.

Table 3: Prevalence of malaria parasitaemia in participants by Microscopy ( $N=264$ )

Variables positive negative prevalence (\%)

ITNs

$9(8.3)$

99(91.7)

$8.3 \%$

IPT-sp

15(7.9)

$175(92.1)$

$7.9 \%$

INSECTICIDE SPRAY

18(10.8)

148(89.2)

$10.8 \%$

Prevalence of malaria parasitaemia in participants by Microscopy $(\mathrm{N}=264)$

Variables positive negative prevalence (\%)

ITNs $9(8.3) 99(91.7) 8.3 \%$

IPT-sp 15(7.9) 175(92.1) 7.9\%

INSECTICIDE SPRAY 18(10.8) 148(89.2) 10.8\%

There was a statistically significant association (as shown in Table 4) between participants diagnosed with malaria parasitaemia and the use of insecticide spray $(p<0.001)$, ITNs $(p<0.001)$, and IPT-sp $(p<$ $0.001)$. 
Table 4

Association between maternal parasitaemia and use of preventive strategies

\begin{tabular}{|lllllll|}
\hline Variables & \multicolumn{2}{l}{ Maternal parasitaemia } & Total & X2 & df & p-value \\
\cline { 2 - 6 } & Present & Absent & & & & \\
\hline Insecticide spray use & & & & & & \\
\hline Yes & $18(10.8)$ & $148(89.2)$ & $166(100.0)$ & 34.109 & 1 & $<0.001$ \\
\hline No & $41(41.8)$ & $57(58.2)$ & $98(100.0)$ & & & \\
\hline ITN use & & & & & & \\
\hline Yes & $9(8.3)$ & $99(91.7)$ & $108(100.0)$ & 20.687 & 1 & $<.001$ \\
\hline No & $50(32.1)$ & $106(67.9)$ & $156(100.0)$ & & & \\
\hline IPT-SP & & & & & & \\
\hline Yes & $15(7.9)$ & $175(92.1)$ & $190(100.0)$ & 81.600 & 1 & $<.001$ \\
\hline No & $44(59.5)$ & $30(40.5)$ & $74(100.0)$ & & \\
\hline
\end{tabular}

Logistic regression analysis (Table 5 ) showed a statistically significant association between malaria infection rate and use of insecticide spray $(p=0.001)$, ITNs $(p=0.003)$ and IPT-sp $(p<0.001)$.

Table 5

Logistic regression analysis

\begin{tabular}{|c|c|c|c|c|c|}
\hline Variables & B & S.E. & Wald & OR (95\% C.I.) & $P$ value \\
\hline \multicolumn{6}{|c|}{ Insecticide spray use } \\
\hline Yes & -1.438 & 0.387 & 13.789 & $0.237(0.111-0.507)$ & $<0.001$ \\
\hline No & & & & 1 & \\
\hline \multicolumn{6}{|l|}{ ITN use } \\
\hline Yes & -1.342 & 0.456 & 8.653 & $0.261(0.107-0.639)$ & 0.003 \\
\hline No & & & & 1 & \\
\hline \multicolumn{6}{|l|}{ IPT-SP } \\
\hline Yes & -2.638 & 0.387 & 46.464 & $0.072(0.034-0.153)$ & $<0.001$ \\
\hline No & & & & 1 & \\
\hline Constant & 1.407 & 0.344 & 16.703 & 4.084 & $<0.001$ \\
\hline
\end{tabular}


Findings from this study showed that the mode of malaria prevention methods being reported to be effective and adopted for use were IPT-sp, Insecticide sprays and ITNs. This finding is similar to many other studies where IPT-sp was the leading malaria prevention method $[14,15]$. This could be explained as probably being due to the adoption of these methods by the National Policy for the control of malaria in Nigeria in line with WHO guidelines [5]. Although, Insecticide Spray is not a National Policy, its use has become increasingly popular in some countries in sub-Saharan Africa especially in rural settings [16].

The study revealed that ITNs was the least utilized malaria prevention method as only $40.9 \%$ used ITNs. This rate is rather poor considering that this approach has been discovered to play a major role in malaria prevention methods [2,5]. This is because the figure fall short of the Global Malaria Action Plan that targeted $100 \%$ ITNs utilization coverage for pregnant women [5]. The low utilization of ITNs in this study could be due to several factors. High cost of the net and its unavailability, in addition to poor knowledge of its effectiveness by the end users have been linked to factors contributing to the poor utilization of this method especially in rural areas[7, 8]. However, this finding is similar to the $39.1 \%$ obtained from a study in Enugu and $41.1 \%$ from Owerri, Southeastern Nigeria $[10,11]$. This may be due to the similarities in the study design and setting. However, the result was higher than the reported utilization rate in Ibadan [7, 8], Calabar [9] and the National estimate rate of $33.7 \%$ [6] but less than $75,0 \%$ obtained from a study in Sokoto, Northwestern Nigeria [12]. This was because, the national utilization rate was only calculated for participants who slept under an ITNs the night before survey was conducted. The resultant implication of the low ITNs utilization in the study area is that sadly, the possibility of achieving the $100 \%$ utilization coverage as recommended by the Global Action Plan is very low. The finding further revealed that participants who used ITNs as prevention measure against malaria had a lower prevalence (8.3\%) of malaria infection while those who shunned the use of ITNs in the index pregnancy had a higher prevalence $(32.1 \%)$ of malaria infection. This finding is consistent with previous studies in Nigeria which found ITNs to be protective against malaria in pregnancy $[8,11,20]$.

In contrast to the poor usage of ITNs, a large proportion (72.0\%) of the participants in this study received prophylactic drugs (IPT-sp) for malaria. However, the researchers in the present study did not ascertain whether participants had experience quickening prior to receiving IPT-sp, and so, may have underestimated the proportion of participants who received IPT-sp. The high uptake of this method as compared to ITNs could be due to the fact that it is given to the pregnant mother at presentation during the booking clinic in line with WHO guidelines [3, 5]. Nevertheless, this proportion (72.0\%) of IPT-sp use still fall short of $100 \%$ targeted by the global action plan [3,5]. The implications of the low uptake of IPT$\mathrm{sp}$ in this study raises concerns such as how much lower uptake might be in pregnant women who do not access antenatal care. This study revealed that participants who shunned the use of IPT-sp as preventive measure had the highest prevalence (59.5\%) of malaria while participants who indicated the use of IPT-sp had the lowest prevalence (7.9\%) of malaria. This result further proved the effectiveness of IPT-sp. IPT-sp in pregnancy has been established to lower the incidence of maternal anaemia and low birth weight baby through prevention of maternal parasitaemia $[8,9$,$] . The overall result of this study is similar to that$ documented by Inyang-Etoh et al [20]. It was also consistent with previous studies in Nigeria which found Loading [MathJax]/jax/output/CommonHTML/jax.js gnancy [11,16,]. However, a study by Umeh et al showed no 
difference in parasitaemia levels between treatment and control groups but suggested other confounding factors like participant education and socio-economic status as reasons for the low parasitaemia [21].

Similarly, this study revealed that $62.9 \%$ of participants used insecticide sprays as malaria prevention measure. This finding was similar to finding by Tongo et al [15], but higher than the reported finding by Michael et al [16]. This study revealed that women who used insecticide spray had a lower parasitaemia $(10.8 \%)$ than those who did not $(41.8 \%)$. This finding was consistent with the findings of other studies that insecticide spray is an effective control measure against malaria $[11,16]$. Although, the use of insecticide spray is not a National Policy, the result from this study suggested that it is becoming popular among the study population as an effective malaria control measure and if properly implemented, will contribute positively to the control of malaria in endemic areas. However, some studies have shown that insecticide sprays are no longer as effective to combat the new species of mosquitoes that are now rampant in some parts of this country [4]. A study by Joseph et al, showed that women who used insecticide spray have a higher prevalence of malaria infection than those who did not [4].

Finally, this study has demonstrated low prevalence of maternal parasitaemia among the participants who used malaria prevention methods. However, majority of the participants in this study use one or both forms of malaria preventive measures, but its use does not offer $100 \%$ panacea against malaria infection. This is because the women cannot remain indoors for almost 24 hours as ITNs and Insecticide sprays offer protection only during the period of use [22].

\section{Limitation:}

The study focused on the protective effectiveness of the three malaria prevention methods that were most commonly employed by the study population. Thus, it is possible that there were other prevention methods that were occasionally practiced by the participants but not included in the questionnaire. Therefore, there may be other con-founding factors that are associated with the level of maternal parasitaemia in this study. Secondly, the study was facility and not community based, therefore, findings from this study may not be a true reflection of the utilization rate of these malaria prevention methods in the community. Also, the questionnaires were interviewer administered, therefore, it is possible that the participants might have introduced some biases in the way they responded to the use of these malaria prevention methods.

Benefit of the study: Interventions were put in place for the participants identified with poor utilization of malaria preventive methods and those with malaria parasitaemia. These interventions included:

1. Detailed health education about the benefits of the usage of the preventive methods was given to the participants in order to improve its use.

2. Those participants with malaria parasitaemia were treated with safe antimalaria drug to prevent the Loading [MathJax]/jax/output/CommonHTML/jax.js en of malaria. 
Implications for the policy makers:

The study revealed that several years after Nigeria has adopted the use of ITNs and IPT-sp for malaria control, the desire goals are yet to be achieved. Therefore, stakeholders in the health sector should strengthen health education in the rural areas to improve utilization of these preventive measures. There is also need for the policy makers to canvass for more funding at the primary health care so as to make ITNs and IPT-sp free and accessible to the end users especially at the rural areas.

\section{Recommendations:}

1. In view of the consequences associated with malaria in pregnancy, there is need for concerted efforts to ensure proper implementation of the national policy as adopted by other countries.

2. Information, education and communication based strategies should be employed to drive home the anti-malaria campaign especially among the pregnant women in the rural areas who are more vulnerable.

3. Large community-based studies are required to clarify fully the utilization and effectiveness of these malaria preventive methods.

4. Further research is needed to identify factors negating the poor utilization of these malaria preventive methods among the study population.

\section{Conclusion}

The study has shown that the utilization of ITNs and IPT-sp are still poor. In view of the consequences associated with malaria in pregnancy, there is need for concerted efforts to ensure proper implementation of the national policy as adopted by other countries. Nevertheless, there is a positive remarkable impact with the use of these malaria prevention methods in pregnancy. The outcome was similar to the findings from previous studies. It can therefore be deduced that the burden of acute malaria among pregnant women and the unborn babies can largely be reduced by the protective effects of these control interventions if properly implemented.

\section{List Of Abbreviations}

ITNs: Insecticide Treated Nets.

IPT-sp: Intermittent Preventive Treatment using sulphadoxine-pyrimethamine

FTH: Federal Teaching Hospital Ido-Ekiti.

\section{Declarations}

Ethical Clearance, Consideration and Consent: Ethical clearance was obtained from the Ethics and Loading [MathJax]/jax/output/CommonHTML/jax.js pspital, Ido-Ekiti. Informed verbal and written consent were 
obtained and participation was fully based on willingness to participate. The participants were free to decline or discontinue anytime without losing any benefit of care or favour to those that participated. The study involved no extra cost to the participants.

Consent for publication: Not Applicable.

Availability of data and materials: The datasets for this study would be made available from the correspondence author on a reasonable request

Declaration of Conflicts of Interest: The authors declare that they have no conflict of interest.

Funding: The research received no specific grant from any funding agency in the public, commercial or not- for-profit sectors

\section{Authors' contribution:}

AOI - Conceptualization of the study, designed the study protocol, data acquisition and analysis and drafted the initial manuscript.

TAA - Literature review, data analysis and review of manuscript for intellectual content.

OMS - Critically revised the protocol for methodological and intellectual content.

EKA - Literature review, review of manuscript for intellectual content

AKA- Literature review, review of manuscript for intellectual content.

OAO. Literature review, review of manuscript for intellectual content.

All authors read and approved the final version of the manuscript prior to submission.

\section{Acknowledgement:}

The authors expressed profound gratitude to the management of the Federal Teaching Hospital Ido- Ekiti for providing conducive atmosphere for the conduct of this research. Appreciation goes to the resident doctors and nurses at the antenatal clinic.

Author's Information: Dr. Ibrahim AO is a medical Consultant and researcher at the Federal Teaching Hospital, Ido-Ekiti, Ekiti State, Southwestern Nigeria. His research interests include malaria prevention and control, emergency care and management, and lifestyle changes in the control of hypertension and diabetes in sub-Saharan Africa.

\section{References}


1. Juliana SD, Nawal MN. Malaria and pregnancy : A Global Health Perspective In : Rev Obstel Gynaecol 2009; 2 (3) : 186-192.

2. WHO. A strategic frame work for malaria prevention and control during pregnancy in the African region. Geneva: WHO; 2004. [Last accessed on feb 5, 2019]. Available from http://www.who.orgAFR/MAL/04/01 [ Last accessed on Feb 5, 2020.]

3. Briand V, Badaut C, Cot M. Placental malaria and infant morbidity. Ann Trop Paediatr 2009; (29): 71 83

4. Joseph OA, Oluwaseun OA, Toluwalase IJ, Olayinka AW, Tuesday O, Adekunle A et al. Malaria in pregnancy: A Demographic and clinical Surveillance at mother and Child Hospital, Ondo, South Western, Nigeria. J Prev Infect Control. 2017; 3 :1

5. World Health Organization/ Global malaria programme. Recent success and key challenges in malaria (internet) 2010. Available from http://www.path.org

6. Wagbatsoma VA, Aigbe EE. Utilization among pregnant women attending antenatal clinic in Etsako West LGA, Edo State, Nigeria. Niger J Clin Pract 2010; 13 : 144-148.

7. Oladokun A, Oladokun RE, Adesina OA. Knowledge and utilization of malaria control measures by pregnant and newly delivered mothers in Ibadan, Nigeria. Afr Health Sci. 2011; 11 (4) : 573-577.

8. Isah AY, Amanabo MA, Ekele BA. Prevalence of malaria parasitaemia amongst asymptomatic pregnant women attending a Nigeria teaching hospital. Annals of African medicine. 2011; 10 (2) : $171-174$

9. Aluko JO, Oluwatosin $\mathrm{AO}$. Utilization of insecticide treated nets during pregnancy among postpartum women, Nigeria. A cross sectional study. BMC Pregnancy Childbirth 2012; $12: 21$

10. Ugwu EO, Ezechukwu PC, Obi SN, Ugwu AO, Okeke TC. Utilization of insecticide treated nets among pregnant women in Enugu, South Eastern Nigeria. Medical and Dental Consultants' Association. 2013; 16 (3): 292-296.

11. Idih EE, Ezem BU, Nzeribe EA, Ohyegbule AO, Duru BC, Amajoyi CC. The relationship between malaria parasitaemia , malaria preventive measures and average birth weight of babies in a tertiary facility in Oweri, Nigeria. Niger Med J. 2016; 57 (4) : 208-212.

12. Naphtali RS, Fana SA. Insecticide Treated Nets Distribution and malaria Infection in Argungu, Nigeria. International Journal of Innovation and Applied Studies 2014; 7(2): 709- 716.

13. Saugare LR, Weiss NS, Brentlinger PE, Richardson BA, Staedke SG, Kiwuwa MS et al. Determinants of use of insecticide treated nets for the prevention of malaria in pregnancy. Jinja, Uganda. PLos One 2012; 7 : e39712.

14. Amase N, Dayo EL, Hellen EA, Aliu AA. Malaria parasitaemia among pregnant women possessing freely donated insecticide treated nets (ITNs) in Ado Ekiti, Nigeria. Global Journal of Biology, Agriculture and Health Sciences. 2014; 3 (1): 86-90

15. Tongo 00 , Orimadegun $A E$, Akinyinka 00 . Utilization of malaria preventive measures during pregnancy and birth outcomes in Ibadan, Nigeria. BMC Pregnancy and childbirth 2011; 11: 60 
16. Michael EF, Amao AO, Akintunde GB, Oluwole OO, Konig W. Use and effects of malaria control measures in pregnancy in Lagos, Nigeria. Korean J Parasitol 2011; 49 : 365-371

17. Ibrahim AO, Bello IS, Shabi OM, Gabriel- Alayode OE, Agboola SM, Elegbede OT. Reliability of Rapid Diagnostic Tests for Malaria among pregnant women in Ido Ekiti, Western Nigeria. Journal of Pharmacy and Pharmacology 2018; 6:825-834

18. Araoye MO. Subject Selection : Sample size determination In: Araoye MO. Research Methodology with Statistics for Health and Social Sciences. Nathadex Publishers; 2003: 117-118.

19. Monif GRG. Infectious disease: In: Obstetrics and gynaecology. Baker DA (ed.) $6^{\text {th }}$ ed. Partenon, New York 2004 : 280-286.

20. Inyang-Etoh EC, Agan TU, Etuk SJ, Inyang-Etoh PC. The role of prophylactic antimalaria in the reduction of placenta parasitaemia among pregnant women in Calabar, Nigeria. Niger Med J. 2011; 52: 235-238.

21. Umeh UA, Obi SN, Onah HE, Ugwu EO, Ajah LO, Umeh CR et al. The impact of intermittent preventive treatment with Sulfadoxine-pyrimethamine on the prevalence of malaria parasitaemia in pregnancy. Trop Doct 2012; 42: 1

22. Ogbodo SO, Nwagha UJ, Okaka ANC, Ogeniyi SC, Okoko RO, Nwagha TU et al. Malaria parasitaemia among pregnant women in a rural community of eastern Nigeria; Need for combined measures. Nigeria Journal of Physiological Sciences 2009; 24 (2):95-100.

\section{Figures}




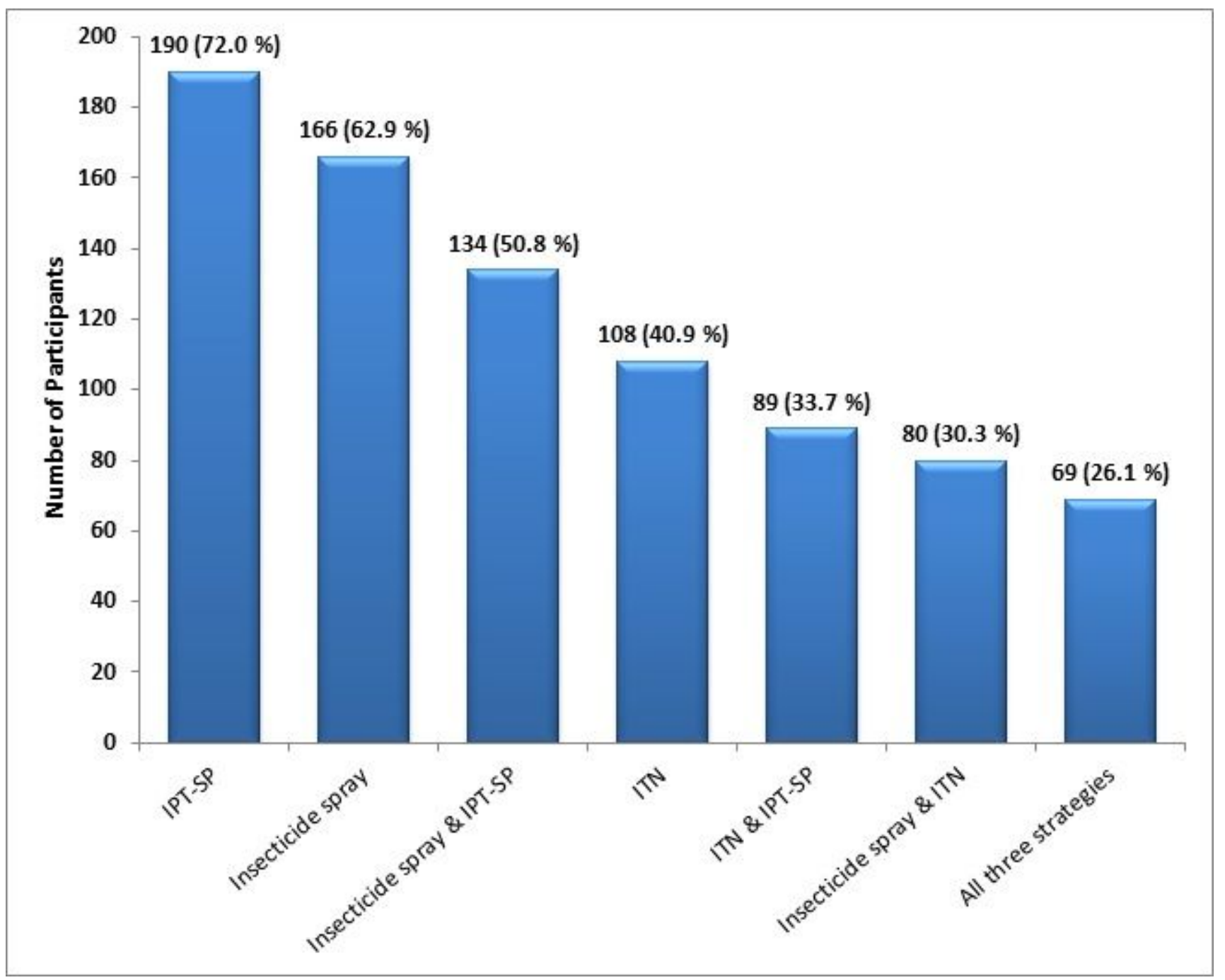

Figure 1

Participants' Utilization of Malaria prevention. 